\title{
Pursuing QCD Phase Transition with Lattice QCD and Experimental Data
}

\author{
Atsushi Nakamura*, \\ RIISE Hiroshima University, Kagamiyama, Higashi-Hiroshima739-8521, Japan \\ E-mail: nakamura@riise.hiroshima-u.ac.jp
}

\section{Keitaro Nagata}

KEK, Oho, Tsukuba305-0801, Japan

E-mail: knagataepost.kek.jp

We propose a new approach to search for the QCD phase transition in the density-temperature plane, in terms of the canonical partition function, $Z_{n}$ s, by combining experimental and lattice numerical studies. We extract the $Z_{n}$ s from the lattice QCD simulations and the RHIC (Relativistic Heavy Ion Collider) data, and construct the moments as a function of $\mu / T$, where $\mu$ and $T$ stand for chemical potential and temperature, respectively.

We calculate also the Lee-Yang zeros of $Z(\xi)$ obtained from the $Z_{n}$ s of the lattice QCD and the RHIC data, where $Z$ and $\xi \equiv \exp (\mu / T)$ are the grand partition function and complex fugacity. We show how to calculate the Lee-Yang zeros with high precision.

As a byproduct, we found clear signals of the Roberge-Weiss transition in the Lee-Yang zero diagram, which occur not only at the pure imaginary chemical potential, but spread into the complex regions with $\operatorname{Im}(\mu)=k \times \frac{2 \pi}{3},(k=1,2)$

31st International Symposium on Lattice Field Theory LATTICE 2013

July 29 - August 3, 2013

Mainz, Germany

\footnotetext{
${ }^{*}$ Speaker.
} 


\section{Introduction}

A dream of researchers in QCD is to find the QCD phase transition and clarify the nature of quark and gluon world. Indeed many interesting phases have been predicted which motivate theoretical and experimental researches [1]. Lattice QCD numerical works are expected to give reliable information as a first principle approach.

So far, however, there have been no conclusive theoretical predictions, nor experimental evidence of the QCD phase transition, once we depart into non-zero baryon density regions. Phenomenological models include ambiguous parameters or applicable limit, and the lattice simulation suffer from the so-called 'sign' problem.

High energy heavy-ion collisions are experiments to explore the QCD phases in $(\mu, T)$ plane, where $\mu$ is the baryon chemical potential, and $T$ is the temperature. The Beam Energy Scan (BES) at RHIC especially targets the QCD phase diagram study [2]. The present work may help to extract information on the QCD phase diagram from the BES.

\section{Constructing $Z_{n}$}

The grand partition function $Z$ and the canonical partition function $Z_{n}$ are related as

$$
Z(\xi, T)=\operatorname{Tr} e^{-(H-\mu \hat{N}) / T}=\sum_{n=-N_{\max }}^{+N_{\max }}\left\langle n\left|e^{-H / T}\right| n\right\rangle e^{\mu n / T}=\sum_{n=-N_{\max }}^{+N_{\max }} Z_{n}(T) \xi^{n},
$$

where $\xi=\exp (\mu / T)$ and

$$
Z_{n}=\left\langle n\left|e^{-H / T}\right| n\right\rangle .
$$

Here, we assume that the number operator $\hat{N}$ commutes with $H$, that is, $\hat{N}$ is a conserved quantity.

\subsection{Lattice}

Lattice numerical simulations evaluate the grand partition function,

$$
Z(\mu, T)=\int \mathscr{D} U(\operatorname{det} \Delta(\mu))^{\mathrm{N}_{\mathrm{f}}} \exp \left(-S_{G}\right) .
$$

where $N_{f}$ is the number of flavor, and in the following we consider $N_{f}=2$ case. The grand partition function, $Z$, can be expanded as a polynomial in terms of the fugacity, $\xi$, with coefficients, $Z_{n}$. Once we realize this relation, we are led to several ways to get $Z_{n}$ from $Z$.

One is to insert the delta function

$$
\delta(n-\hat{N})=\frac{1}{2 \pi} \int d \theta e^{i \theta(n-\hat{N})}
$$

into $Z(T)$, where $\theta=\mu_{I} / T$. Since for the pure imaginary chemical potential, $\mu_{I}$, the fermion determinant is real, and no sign problem appears, we can therefore integrate $Z\left(\mu_{I}\right)$ over $\mu_{I} / T$.

This method is numerically unstable for large $n$ as shown in Ref.[4]. Another method was proposed in Ref.[3], and further investigated in Ref.[5]. The fermion matrix, $\Delta$, is expressed by the reduction formula developed in Ref. [6] as,

$$
\operatorname{det} \Delta(\mu)=C_{0} \xi^{-N_{\mathrm{red}} / 2} \prod_{n=1}^{N_{\mathrm{red}}}\left(\lambda_{n}+\xi\right)=C_{0} \sum_{n=0}^{N_{\mathrm{red}}} c_{n} \xi^{n-N_{\mathrm{red}} / 2}=C_{0} \sum_{n=-N_{\mathrm{red}} / 2}^{N_{\mathrm{red}} / 2} c_{n} \xi^{n},
$$




\begin{tabular}{llc}
\hline$\sqrt{s}(\mathrm{GeV})$ & $\xi$ obtained here & $\xi$ obtained from a freeze-out analysis (Ref.[7]) \\
\hline 11.5 & $7.48331(1)$ & 8.040 \\
19.6 & $3.209(15)$ & 3.623 \\
27 & $2.4342(51)$ & 2.615 \\
39 & $1.883(12)$ & 1.981 \\
62.4 & $1.5338(3)$ & 1.551 \\
200 & $1.17497(9)$ & 1.152 \\
\hline
\end{tabular}

where $\lambda$ 's are eigen values of the reduced matrix. where $N_{\text {red }}=4 N_{c} N_{x} N_{y} N_{z}, N_{c}$ is the number of colors, and $N_{x}, N_{y}$ and $N_{z}$ denote the lattice spatial size. A lattice size is $8^{3} \times 4$ and $10^{3} \times 4$, and we consider a heavy quark case, $m_{\pi} / m_{\rho}=0.8$.

In Eq. (2.3), we set

$$
\operatorname{det} \Delta(\mu) \rightarrow(\operatorname{det} \Delta(\mu) / \operatorname{det} \Delta(0)) \operatorname{det} \Delta(0),
$$

and use $\operatorname{det} \Delta(0)$ as the integration measure. Inserting Eqs.(2.6) with (2.5) into Eq.(2.3), the canonical partition function, $Z_{n}$ is obtained as a coefficient of $\xi^{n}$.

Since the Monte Carlo update is performed with $\operatorname{det} \Delta(0)$, no sign problem due to the complex fermion determinant exists in this method, but there is an overlap problem especially at low temperature; when averaging $c_{n}$ over generated configurations, its sign fluctuates. i.e., the generated configurations are poor ones from the importance sampling viewpoint.

\subsection{RHIC experiment}

Recent net proton multiplicity measurements have been attracting increasing attention 8 , 9, because they enable the exploration of the QCD phase diagram [10, 11] by varying the colliding energy and observing the evolution trajectory $\left(T, \mu_{B}\right)$ that passes near the critical region in the QCD phase diagram. The net proton multiplicity distributions $P_{n}$ observed in experiments are related to $Z_{n}$ as

$$
P_{n}(\xi)=Z_{n} \xi^{n}
$$

Because of the charge conjugation and parity symmetries, $Z_{n}$

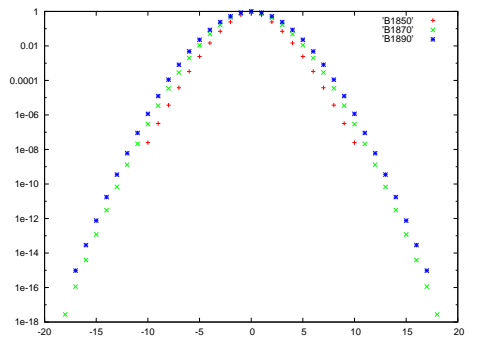
satisfies

$$
Z_{n}=Z_{-n}
$$

Using Eqs. 2.7] and 2.8), we can determine $\xi$ and $Z_{n}$ from the experimental data. Table 2.2 shows the obtained $\xi$ together with that obtained by freeze-out analysis in Ref.[7]. The errors are estimated from the deviations of Eq. (2.8). Note that the freeze-out temperature and the chemical potential in Ref.[7] were obtained from secondary particle distributions and their yields, and no multiplicity is used. 


\section{Moments as a function of $\mu / T$}

Once we get $Z_{n}$, we can construct the grand partition function for arbitrary value of $\xi$,

$$
Z(\xi, T)=\sum_{n=-N_{\max }}^{+N_{\max }} Z_{n}(T) \xi^{n},
$$

Then, at any value of $\xi$, the moments can be calculated as,

$$
\lambda_{k}(\xi)=\left(T \frac{\partial}{\partial \mu}\right)^{k} \log Z=\left(\xi \frac{\partial}{\partial \xi}\right)^{k} \log Z(\xi) .
$$

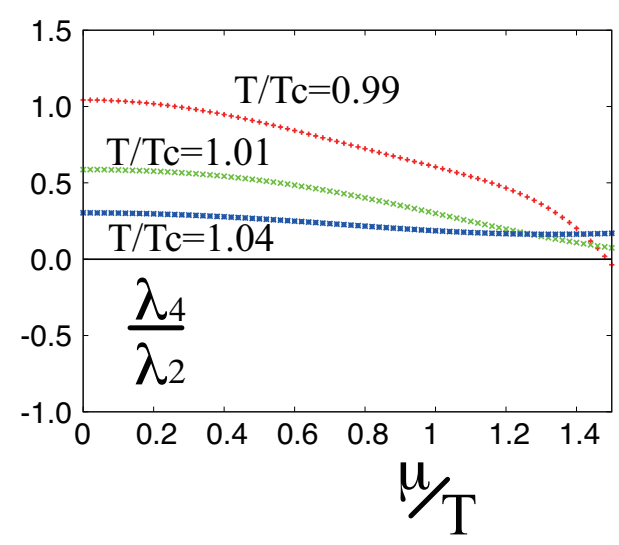

Figure 2: $\lambda_{4} / \lambda_{2}$ as a function of $\mu / T$. (Lattice)

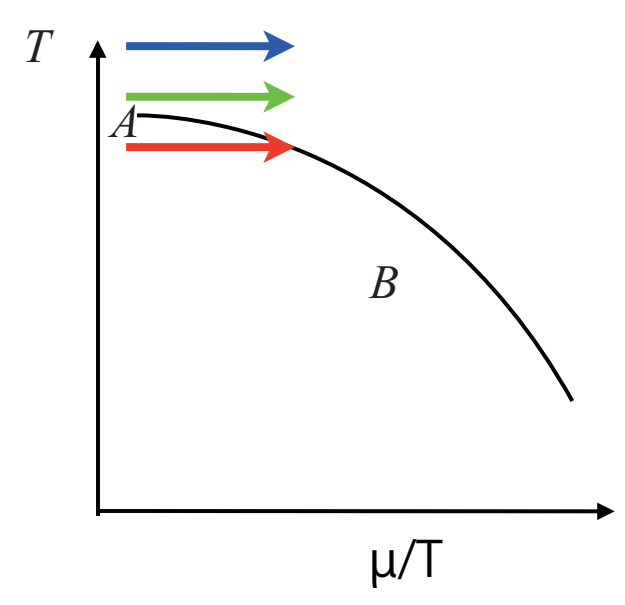

Figure 3: Schematical diagram of the QCD phase in $\mu / T-T$ plane.

In Refs. [12, 13, 14], it is argued that the negative value of $\lambda_{4} / \lambda_{2}$ is a signal of reaching the phase transition. Although the lattice QCD results above $\mu / T>1$ should not be considered too seriously, the behavior of $\lambda_{4} / \lambda_{2}$ in Fig 2 qualitatively matches this picture: Below $T_{c}$, we encounter the phase transition as $\mu / T$ increases (See Fig 3), and $\lambda_{4} / \lambda_{2}$ becomes negative, while above $T_{c}$ we do not see any indication of the phase transition even when we increase $\mu / T$. More realistic lattice simulations with the physical quark masses and large volume will provide a quantitative behaviors.

After such numerical works, it is instructive to see experimental data. We construct $Z_{n}$ from net-proton multiplicity at RHIC[2]. We show $\lambda_{4} / \lambda_{2}$ as a function of $\mu / T$ at $\sqrt{s}=200$ and $19.6 \mathrm{GeV}$, in Figs 3 and 3 respectively. $\sqrt{s}=200 \mathrm{GeV}$ data corresponds to high temperature and low density, while $\sqrt{s}=19.6 \mathrm{GeV}$ is lower temperature and higher density. Therefore the freeze-out points of $\sqrt{s}=200$ and 19.6 locate around $A$ and $B$ in Fig 3 .

\section{Lee-Yang zeros}

Using the equation,

$$
Z(\xi, T)=\sum_{n=-N_{\max }}^{+N_{\max }} Z_{n}(T) \xi^{n},
$$

we can extend $\xi$ from real values to complex values. 


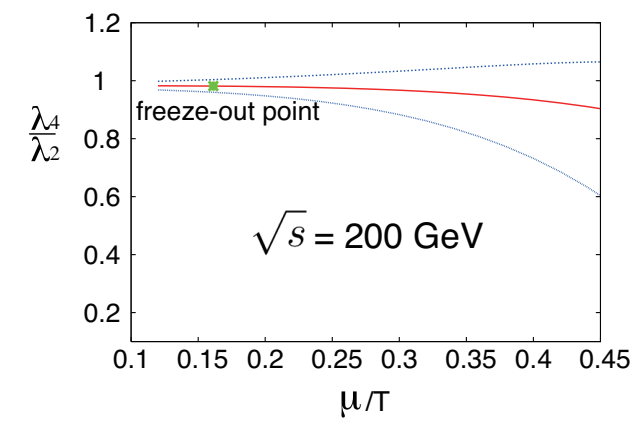

Figure 4: $\lambda_{4} / \lambda_{2}$ as a function of $\mu / T$ constructed from RHIC data (Star) at $\sqrt{s}=200$ $\mathrm{GeV}$. Upper and lower curves stand for estimated errors coming from the ambiguity of $Z_{n}$ of $n \sim N_{\text {max }}$.

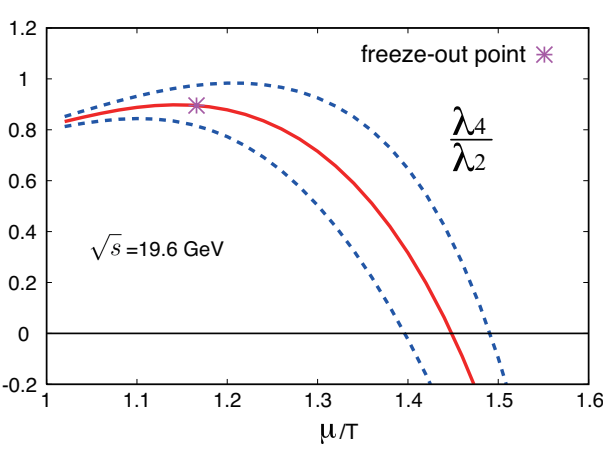

Figure 5: $\lambda_{4} / \lambda_{2}$ as a function of $\mu / T$ constructed from RHIC data (Star) at $\sqrt{s}=19.6$ $\mathrm{GeV}$. Upper and lower curves stand for the same meaning as the previous figure

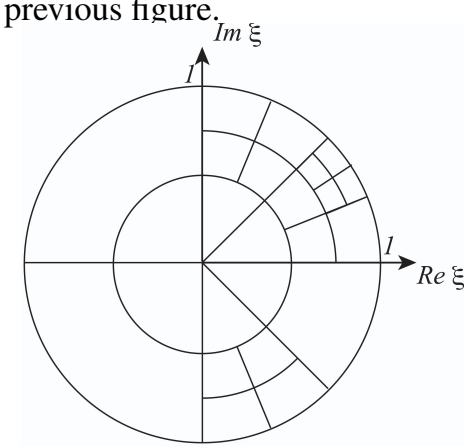

Figure 6: Schematic diagram of CBK contours in the divide-andconquer search for residues.

$f(\xi)=0$ and $Z(\xi)=0$ are equivalent except at the origin, $\xi=0$, where no zeroes exist.

We first map the problem into the calculation of the residue of $f^{\prime} / f$ :

$$
f^{\prime} / f=(\log f)^{\prime}=\sum 1 /\left(\xi-\alpha_{l}\right)
$$

For this equation, the left-hand side is integrated along a contour, and the residues inside the contour are summed according to Cauchy theorem.

All calculations were performed using the multiple-precision package, FMlib[15], and the number of significant digits was $50-100$.

Figs. 7 and 8 show the Lee-Yang zero diagram constructed from RHIC experimental data at $\sqrt{s}=200$ and $19.6 \mathrm{GeV}$. The LYZ at $\sqrt{s}=19.6 \mathrm{GeV}$ distribute much smaller region around the origin than those at $\sqrt{s}=200 \mathrm{GeV}$. It means that the baryon density of the system at $\sqrt{s}=19.6$ $\mathrm{GeV}$ is larger. From the analysis in Sec 3 these data correspond to the location $A$ and $B$ in the phase diagram 3

Although $N_{\max }$ is not sufficiently large in the current experiments, we can see a circle-like structure of the zeros. If this circle crosses the real positive axis as $N_{\max } \rightarrow \infty$, it is a phase transition point. If the system has a crossover transition. the zeros do not cross the axis

As shown in Fig. 6, starting from $0 \leq \theta<2 \pi$ and $0 \leq r \leq 1$ in polar coordinates, the region is divided into four pieces: 


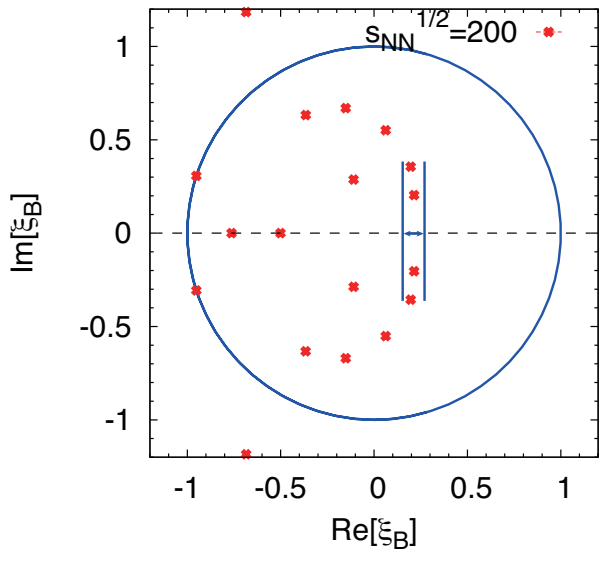

Figure 7: Lee-Yang zero (LYZ) diagram constructed from RHIC data at $\sqrt{s}=200 \mathrm{GeV}$.

$$
\begin{gathered}
\left(\begin{array}{r}
r_{A} \leq r \leq r_{B} \\
\theta_{A} \leq \theta \leq \theta_{B}
\end{array}\right) \quad \rightarrow \\
\left(\begin{array}{cr}
r_{A} \leq r \leq r_{A B} & r_{A} \leq r \leq r_{A B} \\
\theta_{A} \leq \theta \leq \theta_{A B} & \theta_{A B} \leq \theta \leq \theta_{A} \\
r_{A B} \leq r \leq r_{B} & r_{A B} \leq r \leq r_{B} \\
\theta_{A} \leq \theta \leq \theta_{A B} & \theta_{A B} \leq \theta \leq \theta_{A}
\end{array}\right),
\end{gathered}
$$

where $r_{A B}=\left(r_{A}+r_{B}\right) / 2, \theta_{A B}=\left(\theta_{A}+\right.$ $\left.\theta_{B}\right) / 2$. The Cauchy integral is evaluated on each section, which has the shape of a cut Baum-Kuchen (cBK). This divide-and-conquer process is iterated. When no residue is found inside a contour, no further divisions are applied to that region.

Fig 9 shows the Lee-Yang zero structure for a lattice simulation at $T / T c \sim 1.2$. It is marvelous that there are clear indication of the Roberge-Weiss phase transition that there are clear indication of the Roberge-Weiss phase transition, at $\arg (\mu / T)=2 \pi(k+1) / 3$, with integer $k$. This strongly suggests that the Roberge-Weiss transition occurs not only at pure imaginary chemical potential region (on the unit circle in the fugacity plane), but also at $\mu / T=\theta_{R}+2 \pi i(k+1) / 3$. The region of $\theta_{R}$ is finite, but we do not know what controls the length of the region.

\section{Concluding remarks}

In this report, we propose an approach to explore the QCD phase diagram using numerical and experimental analyses. A key issue is $Z_{n}$, the canonical partition functions, which can be obtained from high energy nuclear collision data and from lattice QCD calculations.

Once we have $Z_{n}$, we can reconstruct the grand partition functions, $Z(\mu / T, T)$, at any values of $\mu / T$, although we must consider effects of $N_{\max }$. Current maximum values of $|n|$ are restricted because of the experimental statistics and the lattice overlap problem, because $Z_{n}$ dump rapidly as $|n|$ increases. 
In the process to obtain $Z_{n}$ described in Sec $2, \hat{N}$ should be commutable with the Hamiltonian. Net-baryon and net-charge distribution are satisfied with this condition, but the net-proton distribution is not. We expect that from the net-proton multiplicity the net-baryon distribution is reconstructed either by an event simulation or the method in Ref. [19, 20]. The analysis of the netcharge distribution using our method will also bring rich information on the QCD phase diagram.

We developed also a numerical method to calculate the Lee-Yang zeros. This is very stable, and makes zero determination a well-posed problem: factorization algorithm of polynomial zero search is an ill-problem, because small errors at an early stage lead to large errors in the solution. We can even obtain zeros in a specified complex fugacity region. Using the method, we can obtain the Lee-Yang zeros both for experimental and lattice data. Especially we found that the RobergeWeiss transition above $T_{c}$ [21] occurs not only at pure imaginary chemical potential (on the unit circle in the complex fugacity plane), but also they spread with finite real chemical imaginary potential components. See Fig 9

\section{References}

[1] K. Fukushima and T. Hatsuda, Rept. Prog. Phys. 74:014001, 2011.

[2] "Results from the STAR Beam Energy Scan Program”, L. Kumar, Nucl. Phys. A862-863: 125-131 (2011). (arXiv:1101.4310)

[3] I.M. Barbour and A.J. Bell Nucl. Phys. B372 (1992) 385.

[4] X. Meng, A. Li, A. Alexandru, K.-F. Liu, PoS LATTICE2008:032,2008.

[5] K. Nagata et al., Prog. Theor. Exp. Phys. 2012: 1 A103.

[6] K. Nagata and A. Nakamura, Phys. Rev. D82 094027.

[7] J.Cleymans, H.Oeschler, K.Redlich and S.Wheaton Phys. Rev. C73, 034905 (2006)

[8] M.M. Aggarwal et al., Phys. Rev. Lett. 105022302 (2010).

[9] X. Luo, Quark Matter 2012 arXiv:1210.5573, to appear in Quark Matter 2012 Proceedings.

[10] M. A. Stephanov, K. Rajagopal, and E. V. Shuryak. Phys. Rev. Lett. 81, 4816 (1998).

[11] K.Morita et al., arXiv:1211.4703 [hep-ph].

[12] R. V. Gavai and S. Gupta, Phys. Lett. B696 459, 2011.

[13] V. Skokov, B. Friman and K. Redlich, Phys. Rev. C83, 054904,2011.

[14] M. A. Stephanov, Phys. Rev. Lett. 107, 052301 (2011).

[15] David M. Smith, http://myweb.lmu.edu/dmsmith/FMLIB.html

[16] B. Friman et al., Euro. Phys. J C(2011) 71: 1694.

[17] C. N. Yang and T. D. Lee, Phys. Rev. 87, 404 (1952), T. D. Lee and C. N. Yang, Phys. Rev. 87 410(1952).

[18] K. Nagata and A. Nakamura, Phys. Rev. D83 114507.

[19] M. Kitazawa and M. Asakawa, Phys. Rev. C85, 021901(R) (2012).

[20] A. Bzdak and V. Koch, Phys. Rev. C86, 044904 (2012),

[21] A. Roberge and N. Weiss, Nucl. Phys. B275(1986) 734. 